Received: 04.12 .2017

Revised: 29.12 .2017

Accepted: 26.01 .2018

DOI: $10.17804 / 2410-9908.2018 .1 .006-015$

\title{
ESTIMATED DETERMINATION OF HEAT-AFFECTED ZONES FOR WELDING OF POLYETHYLENE PIPES AT LOW TEMPERATURES
}

\author{
N. P. Starostin ${ }^{\text {a)}}$, O. A. Ammosova ${ }^{\text {b)* }}$ \\ Institute of Oil and Gas Problems, Siberian Branch of the Russian Academy of Sciences, \\ 1 Oktyabrskaya St., Yakutsk, 677891, Russian Federation \\ a) iD https://orcid.org/0000-0002-5686-1817; ${ }^{\text {b) }}$ iD https://orcid.org/0000-0003-3551-0417 冈 ammosova_o@mail.ru \\ *Corresponding author. E-mail: ammosova_o@mail.ru \\ Address for correspondence: ul. Avtodorozhnaya, 20, 677007, Yakutsk, Russian Federation \\ Tel.: +7(4112) 357293
}

The boundaries of heat-affected zones (HAZ) are determined by mathematical modeling of the thermal process of butt welding of polyethylene pipes for gas pipelines. When choosing the process conditions for welding of polyethylene pipes, as well as when investigating the quality of the welded joint, determination of the boundary of the heat-affected zone is of great importance, since structural changes of the welded materials occur in this zone. The mathematical model used takes into account the heat of the phase transition in the temperature range, as well as the thermal effect of burr formed during upsetting. The adequacy of the proposed mathematical model is shown by comparing the experimental and calculated temperature data. The temperature was recorded using a multichannel temperature programmer with a Thermodat-17E3 graphical display. The problem was solved numerically by the finite difference method. The developed algorithms are implemented as a set of programs in the Delphi environment. Numerical simulation was carried out for a $63 \times 5.8$ PE 100 GAZ SDR11 pipe. The admissible area of heat-affected zones is defined. It is formed at admissible air temperatures. The computational experiments have shown the possibility of controlling the temperature regime for welding under conditions of low ambient temperatures and providing the same temperature field variation in the HAZ as at permissible air temperatures. The time of its formation is fixed. By preheating the ends of a welded pipe and using a thermal enclosure weld during cooling at low temperatures, the desired location of the HAZ boundary is achieved.

Keywords: butt welding of polyethylene pipes, gas pipelines, mathematical modeling of thermal process, heat-affected zones, thermal enclosure.

\section{References}

1. Kuliczkowska E., Gierczak M. Buckling failure numerical analysis of HDPE pipes used for the trenchless rehabilitation of a reinforced concrete sewer. Engineering Failure Analysis, 2013, no. 32, pp. 106-112. DOI: 10.1016/j.engfailanal.2013.03.007

2. Luo X., Lu S., Shi J., Li X., Zheng J. Numerical simulation of strength failure of buried polyethylene pipe under foundation settlement. Engineering Failure Analysis, 2015, no. 48, pp. 144-152. DOI: 10.1016/j.engfailanal.2014.11.014

3. Gould S.J.F., Davis P., Beale D.J., Marlow D.R. Failure analysis of a PVC sewer pipeline by fractography and materials characterization. Engineering Failure Analysis, 2013, no. 34, pp. 41-50. DOI: 10.1016/j.engfailanal.2013.07.009

4. Borovsky B.I., Kunsky M.O. Optimization of gas supply systems in residential city districts. Stroitelstvo i Tekhnogennaya Bezopasnost, 2014, no. 50, pp. 29-33. (In Russian).

For citation: Starostin N. P., Ammosova O. A. Estimated determination of heat-affected zones for welding of polyethylene pipes at low temperatures // Diagnostics, Resource and Mechanics of materials and structures. - 2018. Iss. 1. - P. 06-15. - DOI: 10.17804/2410-9908.2018.1.006-015. 
5. Petrishin A. K voprosu ispolzovaniya polietilena $\mathrm{v}$ truboprovodakh. In: Materialy mezhdunarodnoy nauchno-prakticheskoy konferentsii "Nauka segodnya: zadachi $i$ puti ikh resheniya”, Russia, Vologda, 31 May, 2017 [Science Today: Problems and Ways of Solving Them: Proceedings of the International Scientific and Practical Conference]. Tyumen, Marker LLC Publ., 2017, pp. 31-32. ISBN: 978-5-906850-51-5. (In Russian).

6. Lee B.Y., Kim Y.K., Hwnag W.G., Kim J.S., Lee S.Y. Improvement of butt-welding characteristics of double wall polyethylene pipes. Metals and Materials International, 2012, vol. 18, no. 5, pp. 851-856. DOI: 10.1007/s12540-012-5016-5

7. Panaskar N., Terkar R. Study of joining different types of polymers by friction stir welding. In: Mandal D.K., Syan C.S., eds. CAD/CAM, Robotics and Factories of the Future, Ser. Lecture Notes in Mechanical Engineering, Springer, New Delhi, 2016, pp. 731-739. DOI: 10.1007/ 978-81-322-2740-3_70

8. SP 40-102-2000. Svod pravil po proektirovaniyu i stroitelstvu. Proektirovanie i montazh truboprovodov system vodosnabzheniya i kanalizatsii iz polimernykh materialov. Obshchie trebovaniya [Design and Assembly of Polymer Pipelines for Water-Supply and Sewage Systems. General Requirements: Handbook of Instructions]. (In Russian).

9. Starostin N.P., Ammosova O.A. Simulation of the Thermal Process of Butt Welding of Polyethylene Pipes at Low Temperatures. Journal of Engineering Physics and Thermophysics, 2016, vol. 89, iss. 3, pp. 714-720. DOI: 10.1007/s10891-016-1430-8

10. Rodionov A.K., Babenko F.I., Kovalenko N.A. Crack resistance of welded butt joints in polyethylene pipes. Materialy. Tekhnologii. Instrumenty, 2003, vol. 8, no. 3, pp. 19-20. (In Russian).

11. Lai H.S., Tun N.N., Kil S.H., Yoon K.B. Effect of defects on the burst failure of butt fusion welded polyethylene pipes. Journal of Mechanical Science and Technology, 2016, vol. 30, no. 5, pp. 1973-1981. DOI: 10.1007/s12206-016-0403-3

12. Tariq F., Naz N., Khan M.A., Baloch R.A. Failure analysis of high density polyethylene butt weld joint. Journal of Failure Analysis and Prevention, 2012, vol. 12, no. 2, pp. 168-180. DOI: $10.1007 / \mathrm{s} 11668-011-9536-\mathrm{y}$

13. Zakar F., Budinski M. Fracture of a saddle fusion (weld) joint in high density polyethylene (HDPE) pipe. Engineering Failure Analysis, 2017, vol. 82 pp. 481-492. DOI: 10.1016/ j.engfailanal.2017.03.009

14. Avdonin N.A. Matematicheskoe opisanie protsessov kristallizatsii [Mathematical Description of Crystallization Processes]. Riga, Zinatne Publ., 1980, 180 p. (In Russian).

15. Vabishchevich P.N. Chislennye metody resheniya zadach so svobodnoy granitsey [Numerical Methods for Solving Problems with a Free Boundary]. Moscow, MGU Publ., 1987, 164 p. (In Russian).

16. Babichev A.P., Babushkina N.A., Bratkovsky A.M., etc. Fizicheskie velichiny: spravochnik [Physical Quantities: Reference Book, I.S. Grigoriev, E.Z. Meylikhov, eds.]. Moscow, Energoatomizdat Publ., 1991, 1232 p. (In Russian).

For citation: Starostin N. P., Ammosova O. A. Estimated determination of heat-affected zones for welding of polyethylene pipes at low temperatures // Diagnostics, Resource and Mechanics of materials and structures. - 2018. Iss. 1. - P. 06-15. - DOI: 10.17804/2410-9908.2018.1.006-015. 
Подана в журнал: 04.12.2017

УДК 621.791.461

DOI: $10.17804 / 2410-9908.2018 .1 .006-015$

\title{
РАСЧЕТНОЕ ОПРЕДЕЛЕНИЕ ГРАНИЦ ЗОН ТЕРМИЧЕСКОГО ВЛИЯНИЯ ПРИ СВАРКЕ ПОЛИЭТИЛЕНОВЫХ ТРУБ В УСЛОВИЯХ НИЗКИХ ТЕМПЕРАТУР
}

\author{
Н. П. Старостин ${ }^{\text {a) }}$, О. А. Аммосова ${ }^{\text {б)* }}$ \\ ФГБУН Институт проблем нефти и газа СО РАН, \\ ул. Октябрьская, д. 1, Якутск, Российская Федерация
}

a) (iD https://orcid.org/0000-0002-5686-1817; ${ }^{\text {b) }}$ (D) https://orcid.org/0000-0003-3551-0417 冈ammosova_o@mail.ru

\author{
*Ответственный автор. Электронная почта: ammosova_o@mail.ru \\ Адрес для переписки: 677007, г. Якутск, ул. Автодорожная, 20, Российская Федерация \\ Тел.: +7 (4112) 35-72-93
}

На основе математического моделирования теплового процесса при сварке полиэтиленовых труб встык для газопроводов определяют границы зон термического влияния (3ТВ). При выборе технологических режимов сварки полиэтиленовых труб, а также при исследовании качества сварного соединения большую роль имеет определение границы зоны термического влияния, поскольку в ней происходят структурные изменения свариваемых материалов. Используемая математическая модель учитывает теплоту фазового перехода в интервале температур, а также тепловое влияние образующегося при осадке грата. Показана адекватность предлагаемой математической модели путем сопоставления экспериментальных и расчетных температурных данных. Регистрация температур производилась с помощью многоканального программного регулятора температуры с графическим дисплеем ТЕРМОДАТ-17Е3. Поставленная задача решалась численно методом конечных разностей. Разработанные алгоритмы реализованы в виде комплекса программ в среде «Delphi». Численное моделирование проводилось для трубы марки ПЭ 100 ГАЗ SDR11 $63 \times 5,8$. Определяется допустимая область 3ТВ, сформированная при допустимых температурах воздуха, и время ее формирования. Вычислительными экспериментами показана возможность регулирования температурным режимом при сварке в условиях низких температур окружающего воздуха и обеспечения в зоне термического влияния такого же изменения температурного поля, что и при допустимых температурах воздуха. Проведение сварки полиэтиленовых труб с предварительным подогревом и выравниванием температуры, а также проведение этапа осадки свариваемых труб в теплоизоляционной камере с расчетными размерами, приводят к допустимому темпу охлаждения и формированию границы ЗТВ в пределах допустимой области.

Ключевые слова: сварка полиэтиленовых труб встык, газопровод, моделирование теплового процесса, зона термического влияние, теплоизоляционная камера.

\section{1. Введение}

Полиэтиленовые трубы наиболее часто используются для распределительных систем газоснабжения и в других объектах [1-3]. Это связано с главными преимуществами этих труб (прочность, гибкость и химическая инертность) по сравнению с металлическими материалами, такими как, например, стали $[4,5]$. Существуют различные способы соединения полиэтиленовых труб: стыковая, раструбная, электромуфтовая, сварка вибрацией и трением $[6,7]$.

For citation: Starostin N. P., Ammosova O. A. Estimated determination of heat-affected zones for welding of polyethylene pipes at low temperatures // Diagnostics, Resource and Mechanics of materials and structures. - 2018. Iss. 1. - P. 06-15. - DOI: 10.17804/2410-9908.2018.1.006-015. 
При этом с ростом протяженности газопроводов из полиэтиленовых труб крайне важным становится решение проблемы их оперативного ремонта при аварийных ситуациях, особенно в зимний период в северных регионах России. Действующие нормативные документы позволяют проводить сварочные работы при монтаже и ремонте при температуре атмосферного воздуха не ниже $-15{ }^{\circ} \mathrm{C}$ [8]. Назовем интервал температур от -15 до +40 допустимым диапазоном. При более низких температурах воздуха сварочные работы рекомендуется проводить в легких отапливаемых конструкциях с поддержанием температуры из допустимого диапазона. Между тем, такая сварка обусловлена большими энергетическими, непроизводственными затратами и длительной подготовкой, что неприемлемо в аварийных ситуациях.

Исследование теплового процесса сварки полиэтиленовых труб встык для газопроводов при температурах воздуха ниже нормативных приведено в работе [9]. Вычислительными экспериментами показана возможность регулирования температурным режимом при сварке в условиях низких температурах окружающего воздуха и обеспечения в зоне термического влияния такого же изменения температурного поля, что и при допустимых температурах воздуха. Это возможно при проведении предварительного подогрева свариваемых торцов труб, выравнивания температуры, которые предшествуют этапу нагрева (оплавления), как при допустимых температурах воздуха и охлаждении сварного соединения в теплоизоляционной камере. Продолжительности предварительного подогрева, выравнивания и размеры камеры определяются расчетным путем.

\section{2. Постановка задачи и методы решения}

При выборе технологических режимов сварки полиэтиленовых труб, а также при исследовании качества сварного соединения большую роль имеет определение границы зоны термического влияния (ЗТВ), в которой происходят структурные изменения свариваемых материалов. Обычно ЗТВ определяется экспериментально путем исследования структурных показателей. Принимая часто используемое предположение о том, что структурные изменения происходят при температурах выше температуры размягчения материала [10], теоретически определяются границы зон термического влияния при различных режимах сварки. Несоблюдение технологии сварки и отсутствие достаточного технического обслуживания приводят к возникновению дефектов в зоне сварного соединения [11-13].

Поскольку в полиэтилене не существует четко выраженной границы фазового перехода (фазовый переход происходит в интервале температур), то в математической модели необходимо учитывать промежуточную фазу между твердым и жидким веществом, в которой вещество находится как в твердом, так и в жидком состоянии [14]. Границы промежуточной фазы определяются температурами солидуса $T_{S}$ и ликвидуса $T_{L}$.

Температурное поле в стенке трубы в этом случае определяется из уравнения теплопроводности с эффективным коэффициентом теплоемкости $[14,15]$ :

$$
\begin{gathered}
\tilde{C}(T) \frac{\partial T}{\partial t}=\frac{1}{r} \frac{\partial}{\partial r}\left(r \chi(T) \frac{\partial T}{\partial r}\right)+\frac{\partial}{\partial z}\left(\lambda(T) \frac{\partial T}{\partial z}\right) ; \\
0<t \leq t_{m} ; \quad 0<r<r_{\Gamma} ; \quad 0<z<l,
\end{gathered}
$$

где $T(r, z, t)$ - температура в момент времени $t$; $\mathrm{C}(T)$ - удельная теплоемкость; $t_{m}-$ время расчета; $r, z$ - цилиндрические координаты; $\Gamma$ - свободная поверхность грата и части трубы. $\Psi(T)$ - объемная доля твердой фазы; $\rho(T)$ - удельная плотность. Индекс $i=1$ для твердой фазы материала трубы; $i=2$ для жидкой фазы материала трубы.

Эффективный коэффициент теплоемкости вычислим по формуле:

For citation: Starostin N. P., Ammosova O. A. Estimated determination of heat-affected zones for welding of polyethylene pipes at low temperatures // Diagnostics, Resource and Mechanics of materials and structures. - 2018. Iss. 1. - P. 06-15. - DOI: 10.17804/2410-9908.2018.1.006-015. 


$$
\tilde{C}(T)=\left\{\begin{array}{l}
C_{1}, \quad T \leq T_{S} \\
0,5\left(C_{1}+C_{2}\right)+\rho \frac{q(T)}{\Delta}, \quad T_{S}<T<T_{L}, \\
C_{2}, \quad T \geq T_{L}
\end{array}\right.
$$

где $q(T)$ - зависимость теплового потока от температуры, регистрируемая дифференциальным сканирующим калориметром, отнесенная к единице массы вещества; $\Delta=\frac{\left(T_{L}-T_{S}\right)}{\left(t_{2}-t_{1}\right)}-$ скорость нагрева, варьируемая в ДСК; $t_{1}, t_{2}$ - время начала и окончания фазового перехода.

На одном торце задается температура нагретого инструмента:

$$
T(r, 0, t)=T_{H}
$$

Учитывая низкую теплопроводность полиэтилена, примем, что на некотором удалении $\mathrm{z}=l$ от зоны сварки температура трубы не изменяется на протяжении всего времени протекания процесса. Таким образом, на другом торце трубы задается условие:

$$
T(r, l, t)=T_{\text {окр }}
$$

На внешней и внутренней поверхностях трубы происходит конвективный теплообмен с окружающей средой:

$$
\left.\lambda_{i} \frac{\partial T}{\partial r}\right|_{\Gamma}=-\alpha\left(\left.T\right|_{\Gamma}-T_{\text {окр }}\right)
$$

Математически задача сводится к отысканию наиболее удаленной от стыка кривой, в каждой точке которой достигается максимальная температура, равная температуре размягчения материала $80{ }^{\circ} \mathrm{C}$. Поскольку температура в стенке трубы в данном случае представляет функцию трех переменных - радиуса, длины и времени, искомая кривая определяется следующим образом.

Для каждого фиксированного значения радиуса определяется изолиния температуры размягчения, на которой определяется максимальное значение переменной по длине трубы и соответствующее значение времени. Таким образом, пространственная кривая находится в координатах: расстояние от стыка $(z)$, радиус $(r)$ и время $(t)$. Граница ЗТВ определяется как проекция этой кривой на плоскость $z O r$, т. е. граница не зависит от времени, при которой достигнута температура размягчения. Тем не менее, построив проекции найденной кривой на плоскости zOt и rOt, можно получить данные о времени формирования границы 3ТВ.

Численное моделирование проводилось для трубы ПЭ 100 ГАЗ SDR11 63×5,8. Расчеты проводились при следующих данных: внутренний радиус $r_{1}=0,0257$; наружный радиус $r_{2}=0,0315 \mathrm{M} ; \lambda_{1}=0,38 ; \lambda_{2}=0,29 \mathrm{BT} /\left(\kappa \Gamma^{\circ}{ }^{\circ} \mathrm{C}\right) ; \rho_{1}=954 ; \rho_{2}=700 \mathrm{\kappa} / \mathrm{m}^{3} ; c_{1}=1900 ;$ $c_{2}=1900$ Дж/(кг $\left.{ }^{\circ} \mathrm{C}\right)[16]$. Длина подогреваемой области нагревательного инструмента равнялась 3 см. Температура окружающего воздуха 20, минус 15 и минус $40{ }^{\circ} \mathrm{C}$. Шаги по $r$ и $z$ неравномерные, сетка $62 \times 200$, шаг по времени $\tau=1 \mathrm{c}$.

Задача определения нестационарного температурного поля в сварном соединении с учетом фазового перехода в интервале температур решалась численно методом конечных разностей.

Алгоритм сквозного счета строился с использованием чисто неявных схем. Коэффициент теплопроводности в интервале фазового перехода аппроксимировали линейно. Полу-

For citation: Starostin N. P., Ammosova O. A. Estimated determination of heat-affected zones for welding of polyethylene pipes at low temperatures // Diagnostics, Resource and Mechanics of materials and structures. - 2018. Iss. 1. - P. 06-15. - DOI: 10.17804/2410-9908.2018.1.006-015. 
чающиеся при этом нелинейные трехточечные уравнения решались методом итераций, решения на каждой итерации находились методом прогонки. Разработанные алгоритмы реализованы в виде комплекса программ в среде «Delphi».

\section{3. Адекватность модели}

Для подтверждения адекватности математической модели реальному тепловому процессу произведена сварка труб SDR11 63×5,8 при температурах OB ниже нормативных, в ходе которой измерялись температуры в различных точках. Регистрация температур производилась медь-константановыми термопарами $\varnothing 0,1$ мм с помощью многоканального программного регулятора температуры с графическим дисплеем ТЕРМОДАТ-17Е3. Замеры температуры проводились на этапе охлаждения, на котором принята большая часть основных допущений при моделировании теплового процесса.

На рис. 1 приведены результаты сопоставления расчетных и экспериментальных температур в стенке трубы и в камере при сварке полиэтиленовых труб при температуре воздуха $-37{ }^{\circ} \mathrm{C}$. Реальные температуры воздуха в теплоизоляционной камере ниже расчетных. Максимальное отклонение экспериментальных и расчетных зависимостей температуры, составляющее примерно $10{ }^{\circ} \mathrm{C}$, получено в конце рассматриваемого временного интервала. Характер отклонения расчетных и экспериментальных кривых свидетельствует о том, что погрешности расчета температуры воздуха в камере обусловлены недостаточной герметичностью камеры. В начальный период времени расчетные температуры воздуха в камере превышают реальные. Это связано с тем, что в расчетах не учитывается технологическая пауза, составляющая 2-3 с. В то же время эти погрешности также не приводят к высокой погрешности в расчете основного исследуемого параметра - динамики температурного поля в стенке трубы. Результаты сопоставления экспериментальных и расчетных температур показывают адекватность предлагаемой математической модели процесса охлаждения стенки свариваемой трубы с учетом теплового воздействия грата реальному. Таким образом, предлагаемая модель достаточно эффективно может быть использована для регулирования процесса охлаждения.

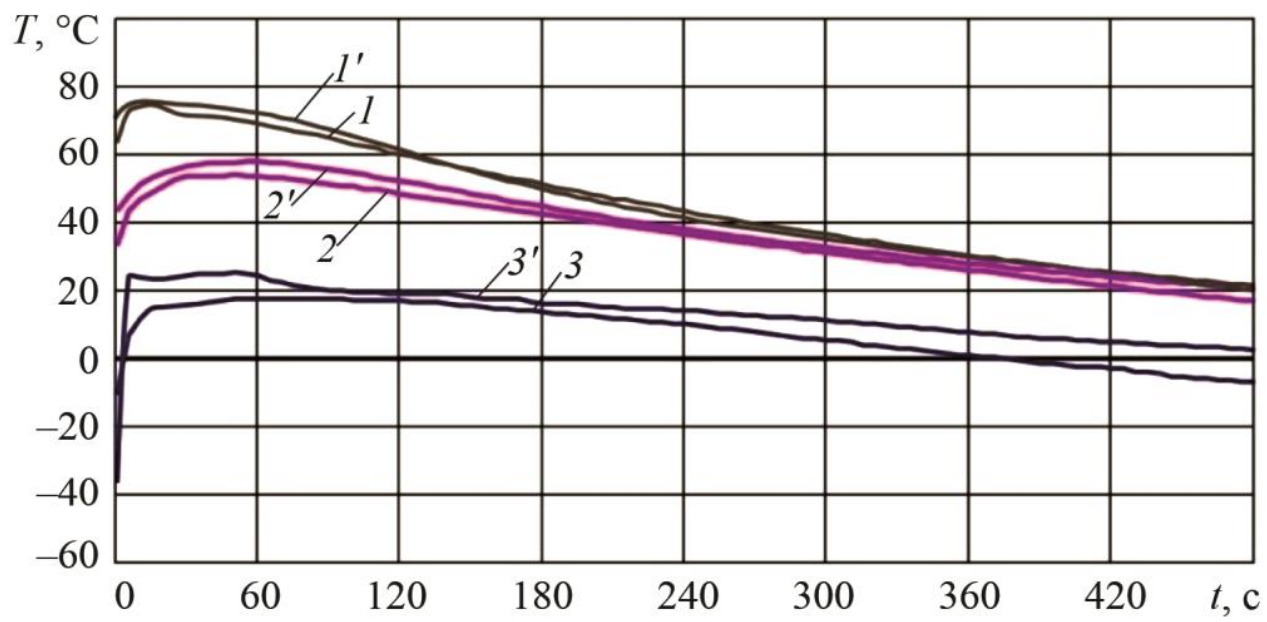

Рис. 1. Зависимости температур по времени в стенке трубы и в камере в следующих точках: $1,1^{\prime}-(0,0283 ; 0,001) ; 2,2^{\prime}-(0,0299 ; 0,003) ; 3,3^{\prime}-$ температура в камере; (1-3) - экспериментальные; (1'-3') - расчетные 


\section{4. Результаты и обсуждение}

При минимально и максимально допустимых температурах воздуха рассчитаны границы 3ТВ, ограничивающие область допустимого местоположения границы (рис. 2 и 3). При этом определено минимальное время ее формирования, которое составляет 30 с после начала этапа осадки, которое назовем допустимым.

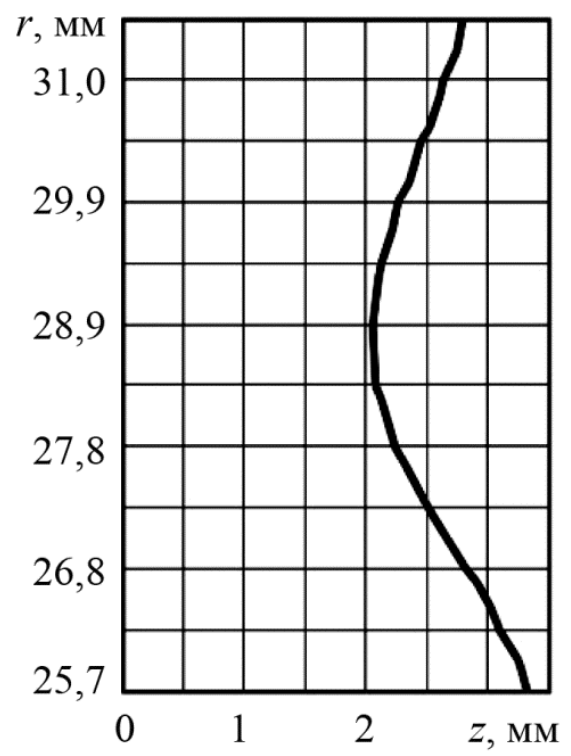

Рис. 2. Зона термического влияния при температуре $\mathrm{OB} 20^{\circ} \mathrm{C}$, период формирования ЗТВ 70 с

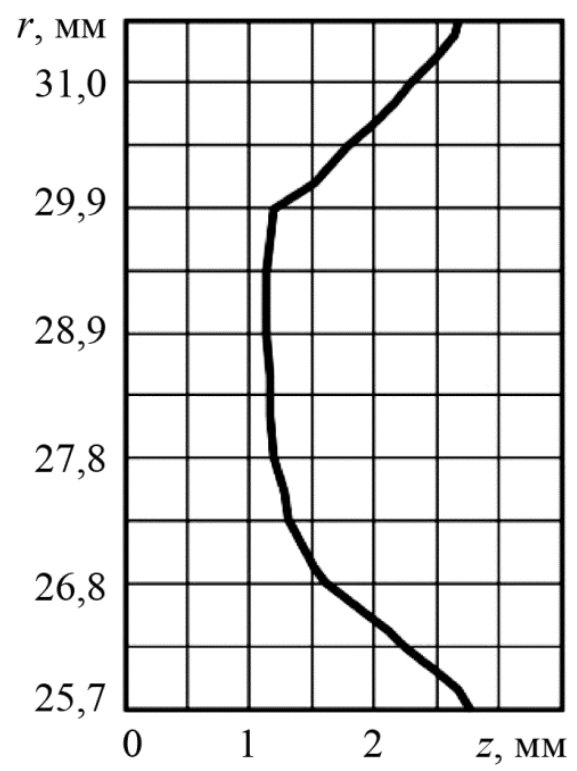

Рис. 3. Зона термического влияния при температуре $\mathrm{OB}-15^{\circ} \mathrm{C}$, время формирования ЗТВ $30 \mathrm{c}$

Определена граница зоны термического влияния, сформированная после нагрева с продолжительностью согласно нормативным документам при температуре воздуха $-40{ }^{\circ} \mathrm{C}$ (рис. 4). При температурах среды ниже нормативных нагрев торцов с продолжительностью, рекомендуемой при минимальной допустимой температуре воздуха, приводит к формированию границы ЗТВ за пределами допустимой области местоположения (рис. 4), при этом пе- 
риод времени ее формирования значительно сокращается. Такое быстрое формирование 3ТВ может оказать влияние на прочность сварного соединения.

Исследования показывают, что при обеспечении одинакового местоположения границы проплавления при низких температурах окружающего воздуха (OB) и охлаждении сварного соединения в теплоизоляционной камере и без нее границы зон термического влияния отличаются незначительно (рис. 5 и 6).

Это свидетельствует о том, что местоположение границы зоны термического влияния в этом случае мало зависит от температуры ОВ. Существенно отличаются периоды времени формирования зон термического влияния, что подтверждает различие темпов охлаждения. Например, время формирования ЗТВ при охлаждении без теплоизоляционной камеры составляет 15 с, что в два раза превышает необходимый темп формирования. В то же время, формирование границы ЗТВ при температуре $\mathrm{OB}-40{ }^{\circ} \mathrm{C}$ с применением теплоизоляционной камеры на этапе осадки происходит за 50 с.

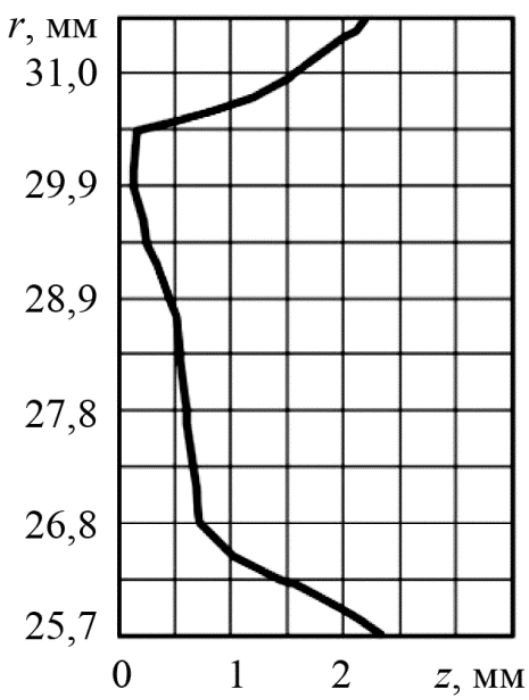

Рис. 4. ЗТВ при сварке ПЭ труб при температуре $\mathrm{OB}-40{ }^{\circ} \mathrm{C}$, период формирования ЗТВ 5 с

Следовательно, отличаться будут также и структуры, и длительные прочности материалов сварного соединения. А это в свою очередь влияет на качество сварного соединения. Поэтому на основании полученных результатов можно предположить, что качество сварного соединения зависит не только от обеспечения необходимой глубины проплавления при нагреве и скорости охлаждения сварного соединения, но и от местоположения границы зоны термического влияния и периода времени ее формирования.

Определение местоположения зоны термического влияния и минимального времени ее формирования при допустимых температурах ОВ позволяет предположить, что соблюдением границы ЗТВ и периода ее формирования при сварке ПЭ труб при температурах ОВ ниже нормативных обеспечивается требуемая прочность сварных соединений.

Проведение сварки полиэтиленовых труб с предварительным подогревом и выравниванием температуры, предложенной в работе, и охлаждение сварного соединения в теплоизоляционной камере с размерами, рассчитанными по методике [9], приводят к допустимому темпу охлаждения и формированию границы ЗТВ в пределах допустимой области.

For citation: Starostin N. P., Ammosova O. A. Estimated determination of heat-affected zones for welding of polyethylene pipes at low temperatures // Diagnostics, Resource and Mechanics of materials and structures. - 2018. Iss. 1. - P. 06-15. - DOI: 10.17804/2410-9908.2018.1.006-015. 


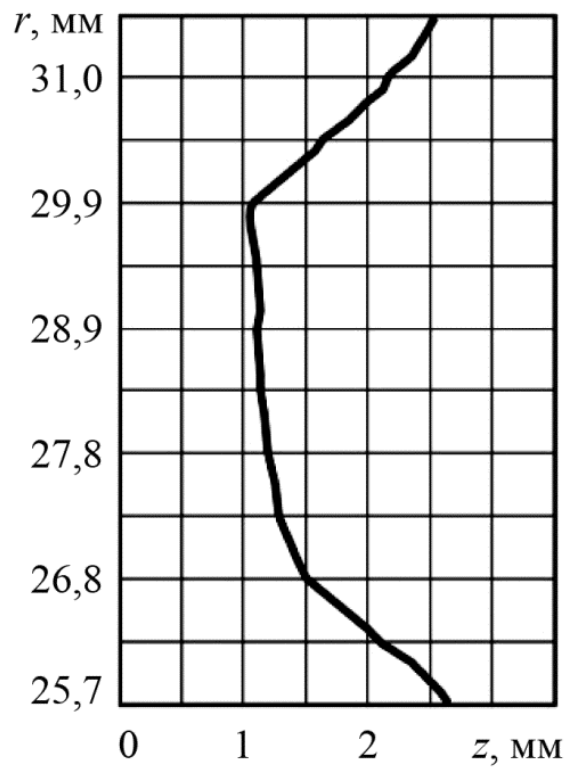

Рис. 5. ЗТВ при сварке ПЭ труб при температуре $\mathrm{OB}-40{ }^{\circ} \mathrm{C}$, продолжительность нагрева 96 с. Охлаждение без камеры.

Период формирования ЗТВ 15 с

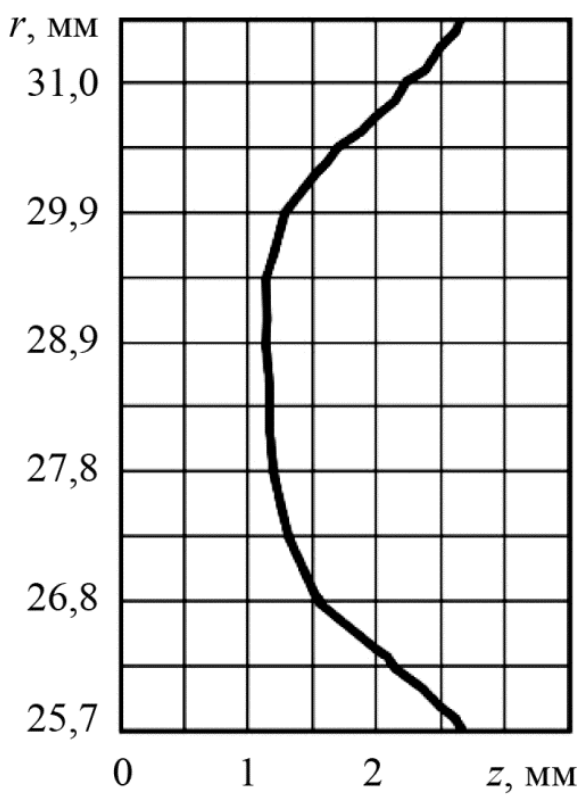

Рис. 6. ЗТВ при сварке ПЭ труб при температуре $\mathrm{OB}-40{ }^{\circ} \mathrm{C}$, продолжительность нагрева 96 с. Охлаждение в теплоизоляционной камере.

Период формирования ЗТВ 50 с

\section{5. Заключение}

Расчетами найдены допустимая граница местоположения зоны термического влияния, сформированная при допустимых температурах воздуха, и время ее формирования. Расчетами показана возможность обеспечения допустимой скорости охлаждения сварного соединения и формирования ЗТВ в требуемых пределах при температурах ОВ ниже нормативных с использованием теплоизоляционной камеры на этапе осадки полиэтиленовых труб. 


\section{Литература}

1. Kuliczkowska E., Gierczak M. Buckling failure numerical analysis of HDPE pipes used for the trenchless rehabilitation of a reinforced concrete sewer. Engineering Failure Analysis. 2013. - No. 32. - P. 106-112. - DOI: 10.1016/j.engfailanal.2013.03.007

2. Numerical simulation of strength failure of buried polyethylene pipe under foundation settlement / X. Luo, S. Lu, J. Shi, X. Li, J. Zheng // Engineering Failure Analysis. - 2015. - No. 48. P. 144-152. - DOI: 10.1016/j.engfailanal.2014.11.014

3. Failure analysis of a PVC sewer pipeline by fractography and materials characterization / S. J. F. Gould, P. Davis, D. J. Beale, D. R. Marlow // Engineering Failure Analysis. - 2013. No. 34. - P. 41-50. - DOI: 10.1016/j.engfailanal.2013.07.009

4. Боровский Б. И., Кунский М. О. Оптимизация систем газоснабжения городских микрорайонов // Строительство и техногенная безопасность. - 2014. - № 50. - С. 29-33.

5. Петришин А. К вопросу использования полиэтилена в трубопроводах // Наука сегодня: задачи и пути их решения : материалы научно-практической конференции, Вологда, 31 мая 2017 г. : сб. науч. тр. - Тюмень : ООО «Маркер», 2017. - С. 31-32. - ISBN: 978-5906850-51-5.

6. Improvement of butt-welding characteristics of double wall polyethylene pipes / B. Y. Lee, Y. K. Kim, W. G. Hwnag, J. S. Kim, S. Y. Lee // Metals and Materials International. - Vol. 18, no. 5. - P. 851-856. - DOI: 10.1007/s12540-012-5016-5

7. Panaskar N., Terkar R. Study of joining different types of polymers by friction stir welding // CAD/CAM, Robotics and Factories of the Future. Ser. Lecture Notes in Mechanical Engineering / ed. by D. K. Mandal, C. S. Syan. - Springer, New Delhi, 2016. - P. 731-739. DOI: $10.1007 / 978-81-322-2740-3 \_70$

8. СП 40-102-2000. Свод правил по проектированию и строительству. Проектирование и монтаж трубопроводов систем водоснабжения и канализации из полимерных материалов. Общие требования.

9. Starostin N. P., Ammosova O. A. Simulation of the Thermal Process of Butt Welding of Polyethylene Pipes at Low Temperatures // Journal of Engineering Physics and Thermophysics. 2016. - Vol. 89, iss. 3. - P. 714-720. - DOI: 10.1007/s10891-016-1430-8

10. Родионов А. К., Бабенко Ф. И., Коваленко Н. А. Трещиностойкость сварных стыковых соединений полиэтиленовых труб // Материалы. Технологии. Инструменты. - 2003. - Т. 8, № 3. - C. 19-20.

11. Effect of defects on the burst failure of butt fusion welded polyethylene pipes / H. S. Lai, N. N. Tun, S. H. Kil, K. B. Yoon // Journal of Mechanical Science and Technology. - 2016. Vol. 30, no. 5. - P. 1973-1981. - DOI: 10.1007/s12206-016-0403-3

12. Failure analysis of high density polyethylene butt weld joint / F. Tariq, N. Naz, M. A. Khan, R. A. Baloch // Journal of Failure Analysis and Prevention. - 2012. - Vol. 12, no. 2. - P. 168-180. DOI: 10.1007/s11668-011-9536-y

13. Zakar F., Budinski M. Fracture of a saddle fusion (weld) joint in high density polyethylene (HDPE) pipe // Engineering Failure Analysis. - 2017. - Vol. 82. - P. 481-492. DOI: $10.1016 / j$.engfailanal.2017.03.009

14. Авдонин Н. А. Математическое описание процессов кристаллизации. - Рига : Зинатне, 1980. $-180 \mathrm{c}$.

15. Вабищевич П. Н. Численные методы решения задач со свободной границей. - Москва : Изд-во МГУ, 1987. - 164 с.

16. Физические величины : справочник / А. П. Бабичев, Н. А. Бабушкина, А. М. Братковский и др. / под ред. И. С. Григорьева, Е. З. Мейлихова. - М. : Энергоатомиздат, 1991. - 1232 с. 